\title{
How to Bridge Gaps in Preventive Care
}

Michelle Winokur, DrPH, and the AfPA Governmental Affairs Team, Alliance for Patient Access (AfPA)

The Alliance for Patient Access (allianceforpatientaccess.org), founded in 2006, is a national network of physicians dedicated to ensuring patient access to approved therapies and appropriate clinical care. AfPA accomplishes this mission by recruiting, training and mobilizing policy-minded physicians to be effective advocates for patient access. AfPA is organized as a non-profit 501(c)(4) corporation and headed by an independent board of directors. Its physician leadership is supported by policy advocacy management and public affairs consultants. In 2012, AfPA established the Institute for Patient Access (IfPA), a related 501(c) (3) non-profit corporation. In keeping with its mission to promote a better understanding of the benefits of the physician-patient relationship in the provision of quality healthcare, IfPA sponsors policy research and educational programming.

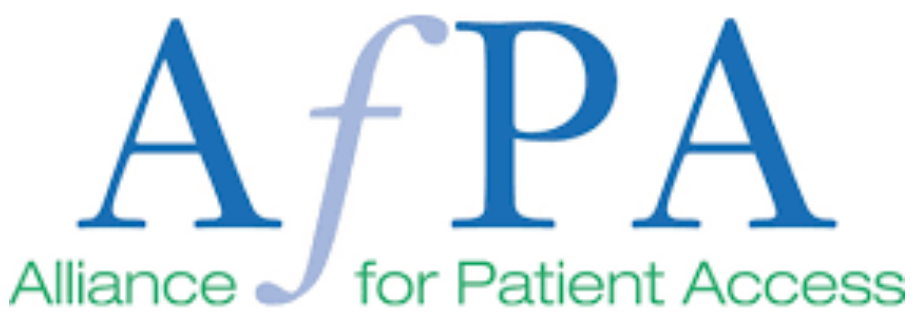

As COVID-19 cases swelled this year, many pediatric specialists saw their daily caseloads dwindle - with vaccination rates tumbling alongside them.

\section{"As COVID-19 cases swelled this year, many pediatric specialists saw their daily caseloads dwindle - with vaccination rates tumbling alongside them."}

The Centers for Disease Control and Prevention reported a significant drop off in provider orders through the Vaccines for Children Program. The program, which provides federally purchased vaccines to approximately half of children, saw roughly 3 million fewer doses of vaccine ordered in April, as compared to $2019 .{ }^{1}$

Child vaccinations were down across the country but declines among the hardest hit urban areas were staggering. New York City, for example, in mid-May reported a "shocking" 91\% decrease in vaccinations for children older than two. ${ }^{2}$ While the downfall in suburban and rural areas was not as severe, millions of children around the country missed routine immunizations. In many cases, immunizations were missed because well-child visits were canceled.

So how can providers and health systems ensure that missed visits, especially those that coincide with immunizations, get made up?

One large health system has a few suggestions. Nemours health system, which operates hospitals and primary care practices in three states, saw a $28.5 \%$ decrease in immunizations among pediatric patients between March and May. (3) Yet they rebounded strong. As recently highlighted in Fierce Healthcare, Nemours is now $4.6 \%$ above last year's total immunization rate for children.

\section{"One large health system has a few suggestions. Nemours health system, which operates hospitals and primary care practices in three states, saw a 28.5\% decrease in immunizations among pediatric patients between March and May. (3)"}

Here's their three-pronged approach to buck the trend:

1. Creating patient cohorts: Nemours separated sick visits from well visits. This was done by time-blocking within single offices so that certain times of the day were reserved for well patients only, while sick patients visited during other hours. In areas with closely located offices, some clinics were earmarked "clean practices." These locations saw only newborns or kids who were not sick.

2. Increasing use of telehealth: Nemours dusted off its underused telemedicine infrastructure. It created a virtual queue that allowed patients to be seen virtually on-demand, "almost like walk-in patients."

3. Outreach to patients: With fewer in-person visits, Nemours reallocated some staff to make outbound calls. After taking the time to explain the system's safety protocols and check in with the family, the staff then helped parents schedule make-up appointments.

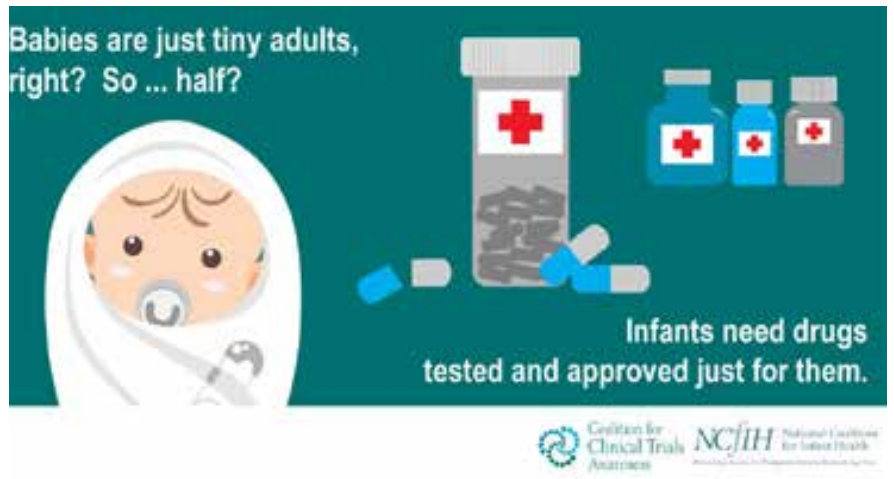


Not all of these approaches - nor the scale to which they were implemented - may not be suitable for every practice. Yet, Nemours' success is a valuable example of how a willingness to adapt and employ some outside-the-box thinking can benefit patients and the public's health in the long term.

\section{"By making adjustments that help}

families feel safe about preventive care, health care providers also can help keep immunizations on track as the COVID-19 pandemic marches on."

Outbreaks of vaccine-preventable diseases are the last thing American communities need right now. By making adjustments that help families feel safe about preventive care, health care providers also can help keep immunizations on track as the COVID-19 pandemic marches on.

The author indicates no disclosures.

\section{References:}

1. Santoli JM, Lindley MC, DeSilva MB, et al. Effects of the COVID-19 pandemic on routine pediatric vaccine ordering and administration - United States, 2020. MMWR Morb Mortal Wkly Rep 2020;69:591-593. DOI: http:// dx.doi.org/10.15585/mmwr.mm6919e2

2. Higgins-Dunn N. New York Mayor de Blasio says number of kids getting vaccinated is down more than $60 \%$. CNBC. May 20, 2020. Accessed Nov 6, 2020. https:// www.cnbc.com/2020/05/20/coronavirus-nyc-mayorsays-number-of-kids-getting-vaccinated-is-down-morethan-60percent.html? source=sharebarlemail\&par=sh arebar

3. Reed T. Many pediatricians say vaccines, visits dropped off amid COVID-19. Nemours says it bucked the trend. Fierce Healthcare. Nov 4, 2020. Accessed Nov 5, 2020. https://www. fiercehealthcare.com/practices/most-pediatricians-say-visits-and-immunizations-have-dropped-offduring-covid-here-s-how

The author has not indicated any disclosures.

NT

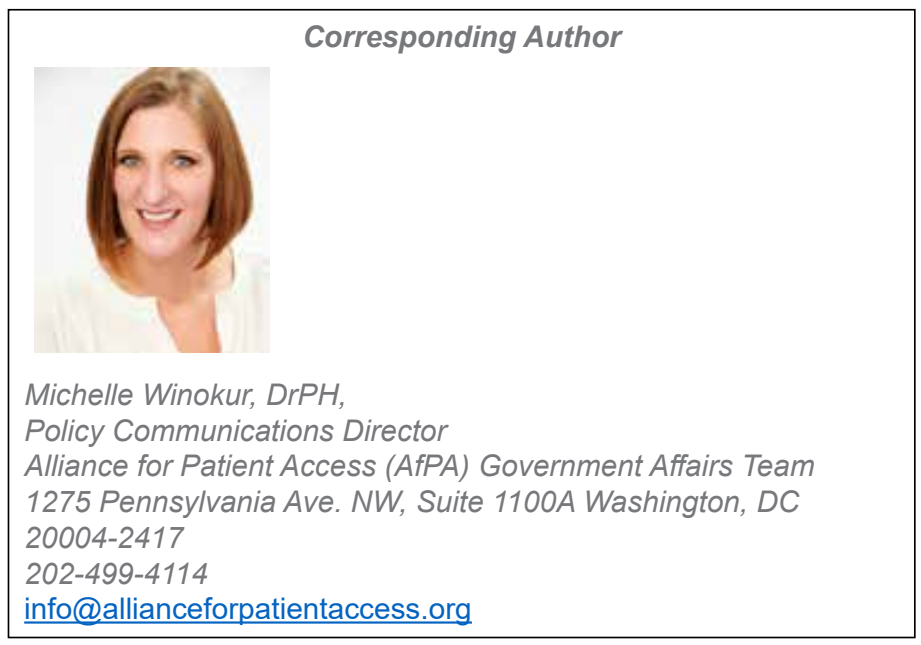

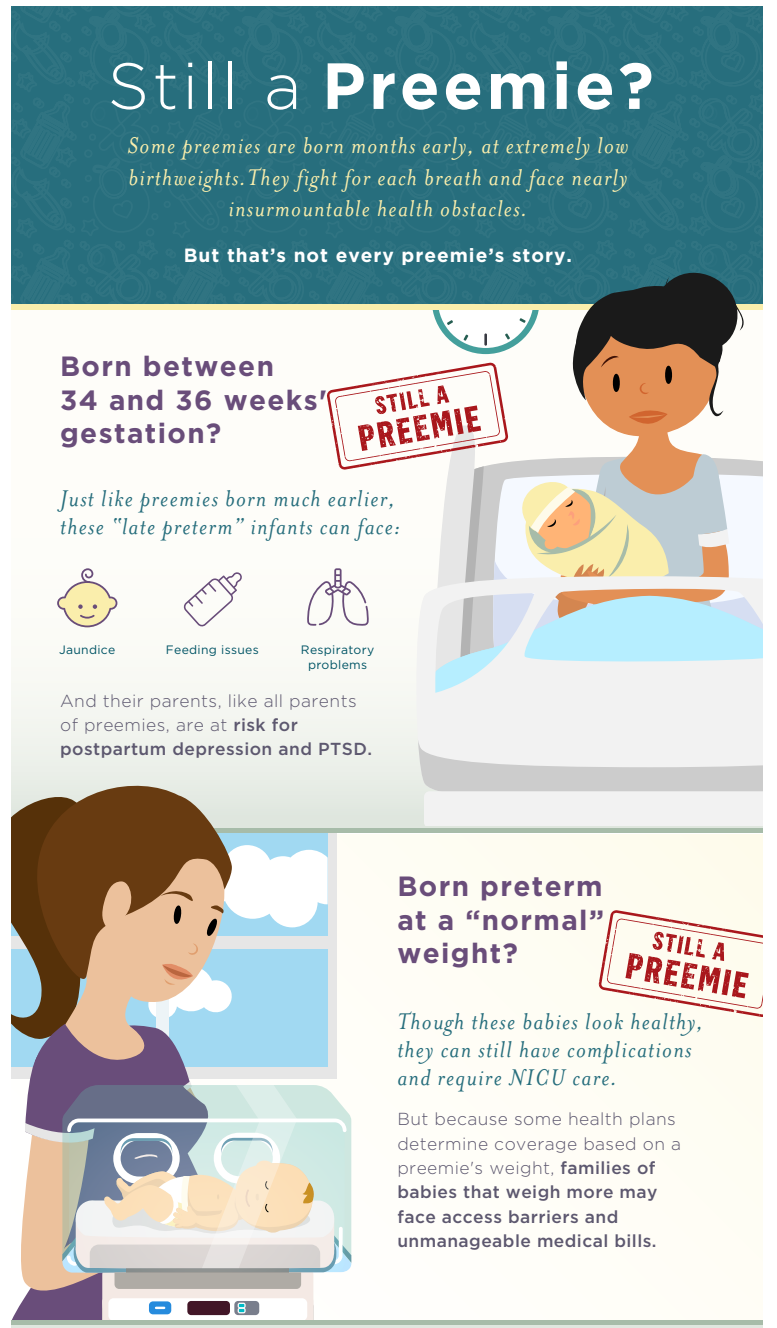

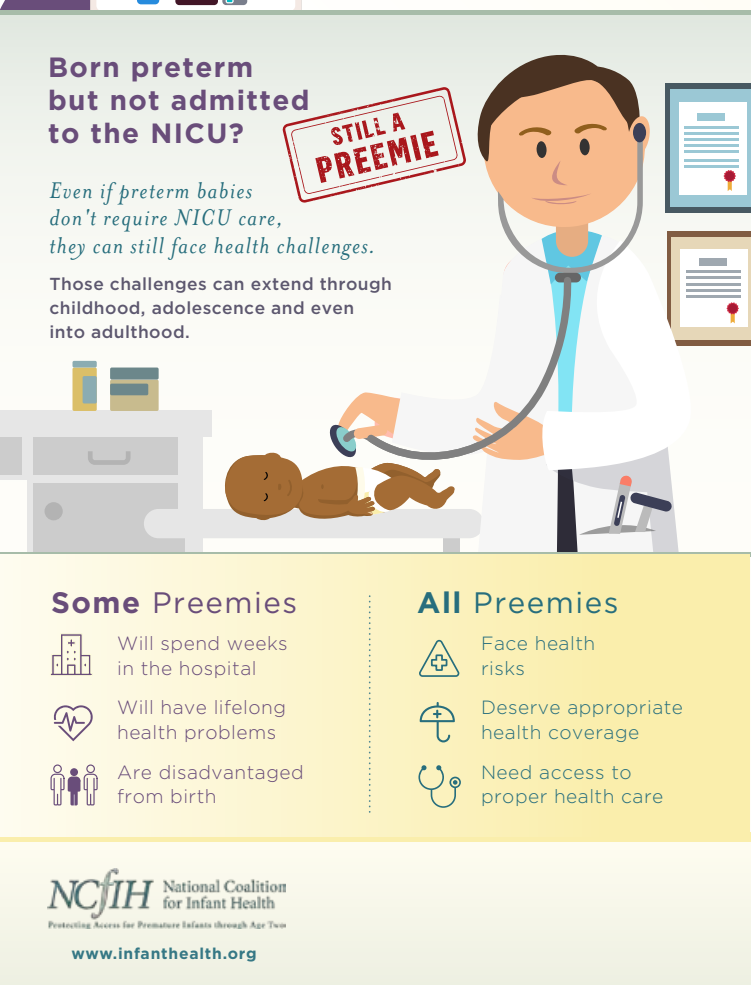

